\title{
Anxiety, Attentional Control, and Performance Impairment in Penalty Kicks
}

\author{
Mark R. Wilson, Greg Wood, and Samuel J. Vine \\ University of Exeter
}

\begin{abstract}
The current study sought to test the predictions of attentional control theory (ACT) in a sporting environment. Fourteen experienced footballers took penalty kicks under low- and high-threat counterbalanced conditions while wearing a gaze registration system. Fixations to target locations (goalkeeper and goal area) were determined using frame-by-frame analysis. When anxious, footballers made faster first fixations and fixated for significantly longer toward the goalkeeper. This disruption in gaze behavior brought about significant reductions in shooting accuracy, with shots becoming significantly centralized and within the goalkeeper's reach. These findings support the predictions of ACT, as anxious participants were more likely to focus on the "threatening" goalkeeper, owing to an increased influence of the stimulus-driven attentional control system.
\end{abstract}

Keywords: soccer, football, visual attention, attentional bias, choking

The influence that anxiety exerts on sporting performance continues to be of major interest to sport psychologists. Various explanations for visuomotor performance impairments caused by increased anxiety have been linked to the disruption of attentional control (see Janelle, 2002, and Wilson, 2008, for reviews). A recent theoretical development from cognitive psychology, attentional control theory (ACT; Eysenck, Derakshan, Santos, \& Calvo, 2007), may therefore provide a useful framework by which to understand such performance disruptions.

ACT is an extension of processing efficiency theory (PET; Eysenck \& Calvo, 1992), which has previously received support in the sport anxiety literature (e.g., Behan \& Wilson, 2008; Murray \& Janelle, 2003, 2007; Nieuwenhuys, Pijpers, Oudejans, \& Bakker, 2008; Williams \& Elliott, 1999; Williams, Vickers, \& Rodrigues, 2002; Wilson, Smith, \& Holmes, 2007a; Wilson, Chattington, Marple-Horvat, \& Smith, 2007b; Wilson, Smith, Chattington, Ford, \& Marple-Horvat, 2006). Whereas PET makes predictions about the effect of anxiety on the general efficiency by which information is processed, ACT is more explicit about the specific attentional processes involved. In this way, the theory shares similarities with other theoretical models of anxiety disorders that propose that anxious individuals both orient more

The authors are with the School of Sport and Health Sciences, University of Exeter, St. Luke's Campus, Exeter, U.K. 
rapidly to anxiety-inducing stimuli, and disengage from them more slowly (see Weierich, Treat, \& Hollingworth, 2008, for a review).

The most general assumption of ACT is that worry increases the allocation of attentional resources to the detection of threat-related stimuli in anxiety-inducing situations (Eysenck et al., 2007). The authors attribute this impairment of attentional control to a disruption in the balance of two attentional systems: a goal-directed (top-down) and a stimulus-driven (bottom-up) attentional system (Corbetta \& Shulman, 2002). Whereas the goal-directed system is influenced by current goals and expectations, the stimulus-driven attentional system responds to salient or conspicuous stimuli. According to ACT, anxiety impairs processing efficiency by reducing attentional control and making it difficult for the goal-directed attentional system to override the stimulus-driven attentional system, especially in the presence of threat-related distracting stimuli (Eysenck et al., 2007).

A recent study by Wilson, Vine, and Wood (2009) tested the predictions of ACT in a sport setting using a basketball free throw task. The authors used the quiet eye period (Vickers, 1996) as a goal-directed measure of attentional control and found that quiet eye durations were significantly reduced in a high-threat condition. Similar to previous studies that have investigated the effects of anxiety on the quiet eye period in far aiming tasks (Behan \& Wilson, 2008; Vickers \& Williams, 2007), this reduction in goal-directed attentional control led to a significant drop in performance effectiveness (reduced free throw percentage accuracy).

Football (soccer) penalty shooting is another example of a visuomotor far aiming skill in which pressure can influence performance. Indeed, anxiety has been reported to be the major contributor to suboptimal performance in penalty kicks (Jordet, 2009; Jordet, Elferink-Gemser, Lemmink, \& Visscher, 2006; Jordet, Hartman, Visscher, and Lemmink, 2007). Jordet et al. (2006) explored professional players' perception of control in penalty taking and found that players who felt a penalty shoot-out to be a "lottery" were more likely to miss than those who believed the outcome was in their own hands. Clearly, the goalkeeper's actions are the principal source of uncertainty bearing on the shooter's success in achieving his or her goal, in what would otherwise be a straightforward aiming task. Uncertainty has been linked with heightened feelings of threat (Fisher \& Zwart, 1982) and increases in cognitive anxiety (Lox, 1992). Given that the goalkeeper is therefore a threatening external stimulus in this evaluative situation, we were interested in determining what effect the goalkeeper might have on the penalty taker's attentional control and performance.

In explaining how anxiety impacts upon attentional control, Eysenck et al. (2007) suggest that, "Threat to a current goal causes attention to be allocated to detecting its source and to deciding how to respond" (p. 338). In the case of penalty shooting, an anxious player may therefore be more likely to attend to the goalkeeper as a source of threat, and attempt to anticipate the goalkeeper's movements. Previous research investigating football penalty performance in experimental settings (e.g., Bakker, Oudejans, Binsch, \& van der Kamp, 2006; Van der Kamp, 2006; Van der Kamp \& Masters, 2008; Wood \& Wilson, in press) has demonstrated that attending to the goalkeeper may be detrimental to performance, as shots are subsequently placed closer within the goalkeeper's reach. Therefore, it has been advised that penalty takers should ignore the actions of the goalkeeper and instead should adopt a top-down attentional strategy, focusing on target-specific locations: the corners of the goal area (Van der Kamp \& Masters, 2008; Wood \& Wilson, in press). 
The influence of anxiety on the visual attention and subsequent performance of penalty takers is therefore of theoretical and practical interest to cognitive sport psychologists. The aim of this research was to discover what effect anxiety has on gaze behavior in a penalty-kicking task and to assess these attentional changes within the theoretical context of attentional control theory (Eysenck et al., 2007). Under ego-threatening conditions, ACT would predict that the goalkeeper-as a salient, conspicuous, and threatening stimulus-would draw the attention of the kicker to a greater extent than under nonthreatening conditions. Such a reduction in the efficiency of attentional control is likely to degrade far aiming performance (as in Wilson et al., 2009). Specifically, it was hypothesized that in the high-threat condition, footballers would fixate earlier (rapid orientation to threat) and for longer (disengage slowly) on the goalkeeper before shooting. This more central, as opposed to distal (target-focused) gaze pattern should result in shots being hit to significantly more central locations (as in Bakker et al., 2006; Wood \& Wilson, in press).

\section{Methods}

\section{Participants}

Fourteen male university standard football players aged 18-22 (mean age, 20.4 years, $S D=1.1$ ) with competitive playing experience ranging from 8 to 16 years (mean, 12.2, SD = 2.0) volunteered to take part in the study. All were right-footed, reported normal vision, and rated their penalty-taking ability to be between 5 and 8 , out of 10 (mean rating of $6.5, S D=1.2$ ). Written consent was gained from all participants and local ethics committee approval was obtained before testing began.

\section{Task Setup}

A standard-sized indoor soccer ball was shot toward a goal that was marked out on an adjacent wall. This target measured $3.6 \mathrm{~m} \times 1.2 \mathrm{~m}$, which is in accordance with regulation indoor soccer goals (JP Lennard, Ltd.; Warwickshire, U.K.), and made up of twelve $30-\mathrm{cm}$ vertical zones that were marked to aid the analysis of shot location. Shots were taken $5 \mathrm{~m}$ from the center of the target, again in accordance with standard indoor football rules. To reduce the potential for injury to the goalkeeper, standard gym mats (32 $\mathrm{mm}$ in thickness) were used to cover the entire kicking area from $1 \mathrm{~m}$ behind the penalty spot to right up against the wall and covering the full width of the goal. This allowed the goalkeeper to dive freely, in safety, while preventing the mats from affecting resultant ball destination.

The same goalkeeper was used throughout the testing period, and he was instructed to try to save each penalty, but not to move before the penalty was struck. He stood directly in the center of the goal with knees bent, arms by his side and hands in front of his body before each shot (see Figure 1). His location and posture were standardized in this way because these variables have been shown to affect performance in penalty taking (Masters, van der Kamp, \& Jackson, 2007; Van der Kamp \& Masters, 2008). These instructions sought to ensure that participants did not attempt to anticipate the goalkeeper's movements, but instead select their own goal-directed target locations. The penalty takers were simply told that the goalkeeper would try and save their penalties and that they should try and shoot to the areas of the goal where they would have the best chance of scoring. 


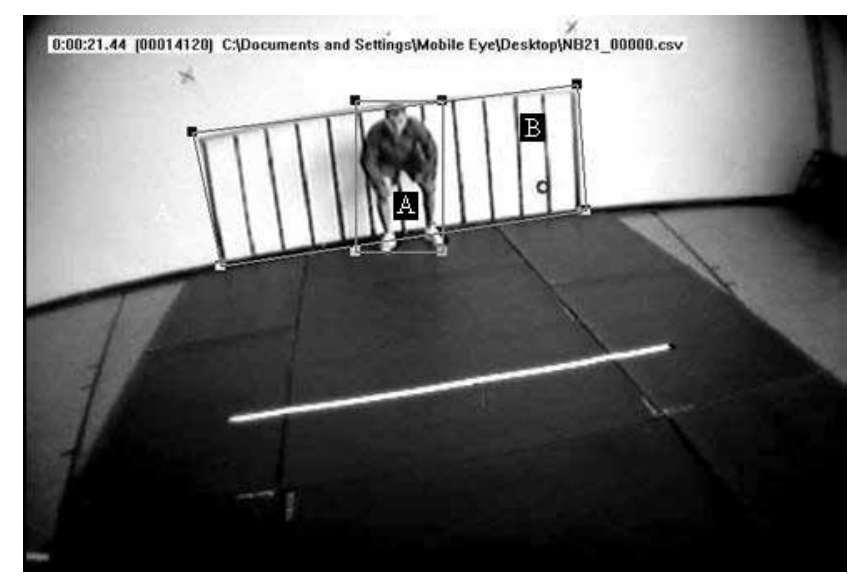

Figure 1 - A screen grab from the GazeTracker software environment showing the experimental setup and the two "LookZones" (areas of interest): the goalkeeper (A) and the goal area (B). The circular cursor, to the goalkeeper's left, represents the participant's point of gaze.

\section{Apparatus}

Participants were fitted with an Applied Science Laboratories Mobile Eye gaze registration system (ASL; Bedford, MA). This system incorporates a pair of lightweight glasses fitted with eye and scene cameras that measure the line of gaze. The system detects the pupil and an infrared corneal reflection in a video image of the eye. The relative position of these features is used to compute visual gaze with respect to the optics. The system incorporates a recording device (a modified digital VCR recording at $25 \mathrm{~Hz}$ ) worn in a waist pouch and was connected to a laptop (Dell; inspiron6400) installed with "Eyevision" (ASL) software. A circular cursor, representing $1^{\circ}$ of visual angle with a $4.5 \mathrm{~mm}$ lens, indicating the location of gaze in a video image of the scene (spatial accuracy of $\pm 0.5^{\circ}$ visual angle; $0.1^{\circ}$ precision) is viewed in real time and recorded for subsequent analysis.

The digital VCR was linked to the laptop via a 10-m FireWire cable, thus permitting near-normal mobility for participants. The researchers and the laptop were located behind and to the left of the participant, to minimize distraction. An externally positioned digital video camera (Canon; MD101) was located $2 \mathrm{~m}$ behind and $4 \mathrm{~m}$ to the right of the participants. This view allowed the penalty spot and the whole target area to be visible.

\section{Design}

Participants performed under low- and high-threat counterbalanced conditions, in a repeated measures research design. In the low-threat condition, nonevaluative instructions were provided to participants, asking them to do their best but stressing that the research was testing the reliability of the calibration of the eye tracker for football tasks. In the high-threat condition, several manipulations were used 
to attempt to ensure that high levels of pressure and ego threat were created (as in Behan \& Wilson, 2008; Murray \& Janelle, 2003). Participants were made aware of a $£ 50$ prize for the kicker with the highest accumulated score. In addition, they were told that a leader board with each participant's name and score would be circulated among all participants. Participants were also told that overall scores would be generated by a computer algorithm developed in a previous study, which analyzed various measures related to shot execution. They were informed that it would be impossible to gauge how well or badly they were doing throughout the trial, so it was best to focus on executing their penalties as they would do normally. This information was introduced to minimize potential reductions in effort and task motivation over successive trials.

\section{Measures}

State Anxiety. The Mental Readiness Form-3 (MRF-3; Krane, 1994) was used to measure state anxiety. This is a shorter alternative to the Competitive State Anxiety Inventory-2 (CSAI-2; Martens, Burton, Vealey, Bump, \& Smith, 1990) with correlations of 0.76 for cognitive anxiety, 0.69 for somatic anxiety, and 0.68 for self-confidence. The MRF-3 has three bipolar 11-point Likert scales that are anchored between worried and not worried for cognitive anxiety, tense and not tense for somatic anxiety, and confident and not confident for self-confidence. This measure was selected as it is a short, expedient inventory that has been employed in other studies testing ACT in sporting environments (e.g., Wilson et al., 2009).

Performance. To minimize the influence of goalkeeper performance on results, a measure of target accuracy was adopted, rather than a measure reflecting goals scored. Target accuracy was measured depending on where on the goal the ball hit, with this location being given a horizontal $(x)$ coordinate (in centimeters), relative to the center of the goal. Each half of the goal consisted of six zones of 30 $\mathrm{cm}$, starting from an "origin" in the center and moving out to $180 \mathrm{~cm}$ at the post (see Figure 1). Higher scores (in centimeters from the central origin) therefore reflected shots that were placed further out of the goalkeeper's reach, where they would have greater chances of scoring (Van der Kamp, 2006).

The coordinate was determined via frame-by-frame analysis of the eye-tracker video file using Quiet Eye Solutions software (www.QuietEyeSolutions.com), with a precision of $5 \mathrm{~cm}$ (one-quarter the diameter of the ball). On 14 occasions, the goalkeeper made a save ( 8 in the no-threat and 6 in the high-threat conditions), in which case the ball did not strike the target area. On these occasions, an estimation of where the ball would have hit was made independently by the second and third authors. Interrater reliability scores were $99.3 \%$

Time to First Fixation on the Goalkeeper. The time taken to orient a first fixation to the goalkeeper from the onset of the trial was measured in seconds using Quiet Eye Solutions software. This measure was designed to reflect early orientation toward threatening stimuli.

Total Fixation Duration. The total (cumulative) fixation durations (in seconds) to each target location (i.e., the goal area and the goalkeeper) in each trial were calculated in the GazeTracker software environment (see Data Analysis). This 
measure was designed to reflect attentional disengagement, that is, the extent to which attention was grabbed and maintained by each location.

Total Number of Fixations. The total number of fixations to each target location in each trial was calculated (see Data Analysis). Whereas longer total fixation duration to an area may indicate delayed disengagement from targets, more fixations to one target location may indicate heightened distractibility to that target.

Preparation Time. The time taken to prepare the shot (trial duration) was calculated in seconds from the external video footage. Trial onset began on the instant the kicker took his hands away from the ball after placing it on the penalty spot and ended on foot-to-ball contact.

\section{Procedure}

Participants attended individually and, after giving their written consent, were told that the aim of the study was to compare kicking performance under different conditions. They familiarized themselves with the testing environment by taking 10 kicks at the target goal with no goalkeeper present. The goalkeeper was excluded at this stage to prevent any previous learning (side preferences, kicking style) occurring for both goalkeeper and kicker. After taking their familiarization kicks, the participants were then fitted with the eye tracker and this was calibrated using each corner of the goal, the center of the goal, and three other points that were marked 1 $\mathrm{m}$ directly above each post and center of the goal. Further quick calibration checks were carried out if the participant felt the eye-tracker glasses had moved or if the researcher noticed any abnormalities on the scene output displayed on the laptop.

Participants were then provided with instructions related to the condition in which they were going to perform, and subsequently completed the MRF-3 before taking four of the seven penalty kicks for that condition. The manipulation instructions were then reiterated and the MRF-3 completed again before the final three kicks were taken. This procedure was adopted to allow a measure of self-reported anxiety to be recorded during, as opposed to just before each testing condition, and to reinforce the anxiety manipulation, which may be diluted over repeated trials (see also Wilson, Smith, et al., 2007a).

\section{Data Analysis}

Point-of-gaze data (consisting of .avi and .csv files) from the Mobile Eye were analyzed using GazeTracker Software (Eye Response Technologies, VA). A "LookZone" (area of interest) was created around the goalkeeper and the goal area, and these were manipulated in a frame-by-frame fashion. This allowed the LookZones to remain stable around the area despite movement in the kicker's visual field (See Figure 1). The software then automatically measured the total number of fixations and each fixation's duration within these preestablished LookZones. Summary statistics for each trial then enabled the total number of fixations and their total (cumulative) fixation duration to be calculated for each LookZone (see Measures). A fixation was classified as three or more consecutive frames $(\geq 120 \mathrm{~ms})$ in which the cursor stayed in the same location (Vickers, 1996). 


\section{Statistics}

MRF-scores, time to fixate on the goalkeeper, preparation time, and performance data were analyzed using paired sampled $t$ tests to explore differences between the conditions. Total fixation duration and the total number of fixations were calculated for each condition and each fixated location of interest (i.e., goalkeeper and goal area). These were then analyzed using a series of fully repeated measures 2 $\times 2$ ANOVAs (Condition $\times$ Location). Significant effects were followed up with Bonferroni-corrected post hoc $t$ tests. Effect sizes were calculated using partial eta squared $\left(\eta_{\mathrm{p}}^{2}\right)$ for omnibus comparisons and Cohen's $d$ for pairwise comparisons (Cohen, 1988).

\section{Results}

Of the 196 shots taken in total, 29 (14.7\%) were excluded owing to technical failure of the eye tracker (11 in the low-threat and 8 in the high-threat condition), or were deliberately excluded because shots missed the target area completely (4 in the low-threat and 6 in the high-threat condition). This left a sample of 167 kicks between low- (83 kicks) and high-threat ( 84 kicks) conditions.

\section{State Anxiety}

There was a significant difference, $t(13)=5.1, p<.01, d=1.11$, in the self-reported cognitive anxiety scores between the low-threat (mean rating of $2.8, S D=1.3$ ) and high-threat (mean rating of $4.6, S D=1.9$ ) condition. Participants reported significantly increased cognitive anxiety in the high-threat condition. Somatic anxiety was also significantly lower, $t(13)=4.9, p<.01, d=0.90$, in the low- (mean rating of $3.4, S D=1.4$ ) as opposed to high-threat condition (mean rating of $4.8, S D=$ 1.7). The reported level of self-confidence did not significantly differ across threat conditions, $t(13)=1.9, p=.07, d=0.54$. However, reported confidence scores were marginally higher (a moderate effect size) in the low- (mean rating of 8.0, $S D=$ 1.2) compared with high-threat (mean rating of $7.3, S D=1.4$ ) condition.

\section{Performance}

There was a significant difference, $t(13)=2.30, p<.05, d=0.78$, in the performance accuracy between low-threat (mean distance of $117.21 \mathrm{~cm}, S D=13.99$ ), and high-threat (mean distance of $103.28 \mathrm{~cm}, S D=21.03$ ) conditions. Shots in the high-threat condition were placed significantly closer to the center of the goal (nearer the goalkeeper) than those in the low-threat condition.

\section{Gaze Behavior / Attentional Control}

Total Number of Fixations. A significant main effect was found for condition, $F(1,13)=8.95, p<.05, \eta_{\mathrm{p}}^{2}=0.41$, with participants making significantly more fixations in the high-threat $(M=13.00, S D=10.02)$ as opposed to the low-threat condition $(M=10.29, S D=10.02)$. No significant main effect was evident for location, $F(1,13)=3.60, p=.08, \eta_{\mathrm{p}}^{2}=0.22$, and the interaction effect was also 
found to be nonsignificant, $F(1,13)=1.57, p=.23, \eta_{\mathrm{p}}{ }^{2}=0.11$. The total number of fixations data are presented in Figure 2.

Total Fixation Duration. A significant main effect was found between conditions, $F(1,13)=6.88, p<.05, \eta_{\mathrm{p}}{ }^{2}=0.35$, indicating that participants spent significantly longer periods of time fixating on both locations under the high-threat $(M=3.37$ $\mathrm{s}, S D=3.27)$ compared with low-threat condition $(M=2.40 \mathrm{~s}, S D=2.29)$. There was a significant main effect for location, $F(1,13)=5.08, p<.05, \eta_{\mathrm{p}}{ }^{2}=0.28$, indicating that participants spent longer fixating on the goalkeeper $(M=3.89 \mathrm{~s}, S D$ $=4.13)$ compared with the goal target area $(M=1.87 \mathrm{~s}, S D=1.87)$. The interaction between condition and location was also found to be significant, $F(1,13)=4.98, p$ $<.05, \eta_{\mathrm{p}}{ }^{2}=0.28$. Post hoc $t$ tests with a Bonferroni correction $(p<.025)$ revealed that total fixation duration to the goalkeeper was significantly longer, $t(13)=2.69$, $p<.025, d=0.41$, in the high-threat $(M=4.73 \mathrm{~s}, S D=4.94)$ as opposed to the low-threat condition $(M=3.04 \mathrm{~s}, S D=3.34)$. A borderline significant difference, $t(13)=2.51, p=.026, d=0.74$, with a moderate-to-large effect size, was found between the total fixation duration between the goalkeeper $(M=4.73 \mathrm{~s}, S D=4.95)$ and goal locations $(M=2.02 \mathrm{~s}, S D=1.61)$, in the high-threat condition. The total fixation duration data are presented in Figure 3.

Time to First Fixation on the Goalkeeper. A paired samples $t$ test revealed that participants were significantly quicker to fixate on the goalkeeper, $t(13)=2.26, p<$ $.05, d=0.34$, in the high-threat $(M=2.45 \mathrm{~s}, S D=0.69)$ compared with low-threat condition $(M=2.68 \mathrm{~s}, S D=0.66)$.

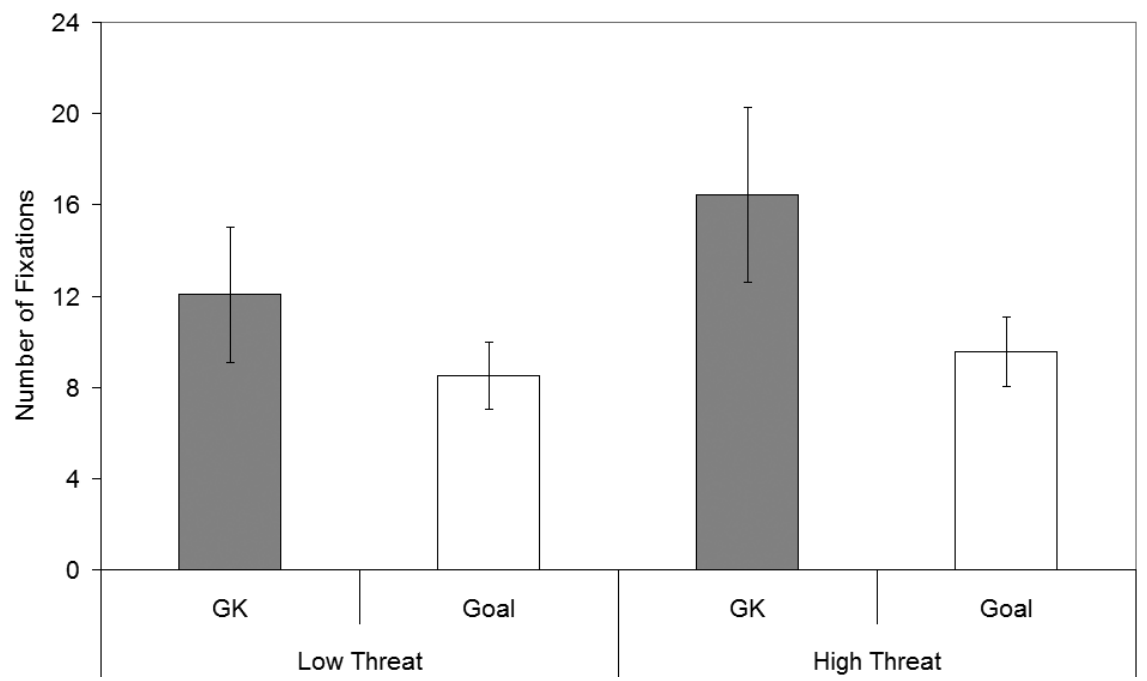

Figure 2 - Gaze behavior data, showing the total number of fixations to each location under low- and high-threat conditions (with standard error of the mean). 


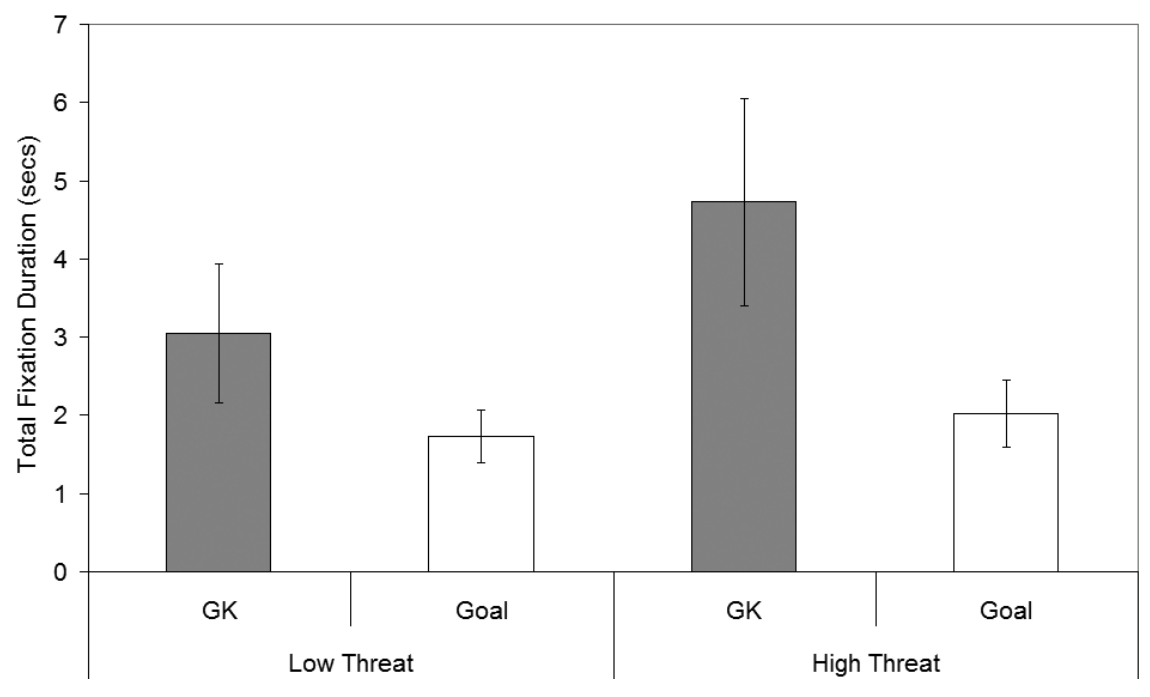

Figure 3 - Gaze behavior data, showing the total fixation duration (in seconds) for each location under low- and high- threat conditions (with standard error of the mean).

\section{Preparation Time}

A paired samples $t$ test revealed that there was no significant difference, $t(13)=$ $.079, p=.44, d=0.09$, in the mean preparation time (trial duration) for each of the seven shots across the low- (mean time of $4.66 \mathrm{~s}, S D=1.17$ ) and high-threat condition (mean time of $4.76 \mathrm{~s}, S D=1.44$ ).

\section{Discussion}

This study aimed to explore anxiety-induced attentional alterations contributing to suboptimal penalty kick performance, using ACT (Eysenck et al., 2007) as an overarching framework. The self-reported anxiety data support the effectiveness of the threat manipulation, although the intensity of threat experienced is almost certainly lower than typically experienced in "real" environments (e.g., penalty shoot-outs; see Jordet et al., 2006, 2007). However, the anxiety levels experienced were similar to those reported in Krane's (1994) validation studies, and other studies using the MRF (e.g., Wilson et al., 2006, 2009). Furthermore, the significant increase in reported anxiety was sufficient to have had a detrimental impact on participants' attentional control (as indexed by gaze behavior) and shooting performance.

\section{Attentional Control}

According to ACT, anxious individuals preferentially attend to conspicuous or salient stimuli at the expense of goal-driven, task-relevant stimuli, owing to the increased influence of the stimulus-driven attentional control system (Eysenck et al., 2007). 
Three gaze measures were adopted to reflect potential differences in the way participants oriented toward, and maintained attention on, the two target locations in both conditions. When participants were anxious, there was a significant increase in the speed at which they first fixated on the goalkeeper, reflecting an attentional bias toward this target. As the goalkeeper adds uncertainty to the outcome of the task from the penalty taker's perspective, the goalkeeper is likely to be considered a source of threat to goal achievement.

The total fixation duration data revealed significant main effects for condition and location and a significant interaction effect (Figure 3). Although both the goalkeeper and goal area were fixated on for longer in the high-threat as opposed to low-threat condition, this effect was more pronounced for the goalkeeper: anxious participants preferentially attended to the goalkeeper. As there was no such interaction effect evident for the total number of fixations to the goalkeeper (Figure 2), these findings suggest that anxiety caused participants to maintain their fixations on the goalkeeper for longer (disengage attention more slowly), as opposed to increasing distractibility. The lack of a significant difference in preparation time (trial length) between conditions, suggests that these changes in gaze measures were due to changes in attentional control and not simply due to participants taking longer in the high-threat condition. Together, the three findings therefore provide support for the predictions of ACT, as an increase in the emphasis of the stimulusdriven attentional system resulted in earlier and longer fixations to the conspicuous, goal-threatening goalkeeper and the utilization of a suboptimal strategy.

There are some caveats concerning our interpretation of the gaze data as being supportive of the predictions of ACT. First, the football penalty task does not provide as elegant a test of the conflict between a goal-driven strategy and stimulus-driven attentional control as a more "pure" cognitive task (e.g., the antisaccade task; Derakshan, Ansari, Hansard, Shoker, \& Eysenck, 2009). Most research examining threat-related attentional biases has adopted protocols using some form of cueing paradigm (see Bar-Haim, Lamy, Pergamin, Bakermans-Kranenburg, \& van IJzendoorn, 2007). This is not possible with a penalty task, as the goalkeeper is located in the same, centralized location for all trials. However, the findings do suggest that the goalkeeper, as a conspicuous and salient stimulus, has preferentially captured attention in the high-threat condition in a manner consistent with ACT's predictions.

Future research could build upon the current study by experimentally manipulating the salience and threat value of the goalkeeper and determining the impact this has on attentional control (as in Derakshan et al., 2009). The more salient and threatening the goalkeeper is, the greater the predicted influence of the stimulus-driven attentional control system. One way in which this salience could be manipulated is by adjusting the goalkeeper's starting position. Although large manipulations may not be ecologically valid, even small changes may impact upon the salience of this distracting stimulus. For example, previous research has demonstrated that even a marginally off-center goalkeeper may influence kick direction (Masters et al., 2007). Furthermore, research by van der Kamp and Masters (2008), examining the effect of the goalkeeper's arm positions while standing (arms by side, or stretched out) found that this affected penalty shot judgments. The goalkeeper could also be made more salient by making him more distracting (e.g., by waving his arms, or jumping on the spot) or by adjusting the color of his shirt. Research in sport settings has found that red shirts confer an advantage, perhaps because the color red has evolved to signify dominance (e.g., Greenlees, Leyland, Thelwell, \& Filby, 2008; Hill \& Barton, 2005). 
The second concern with our interpretation is that, even though there was a significant interaction effect for total fixation duration, participants spent longer fixating on the goalkeeper than on the goal target area irrespective of condition (Figure 3). We expected a clearer interaction effect, with participants fixating for longer on the target goal spaces when not anxious (reflecting top-down, goaldirected attentional control), but becoming more stimulus driven in the threatening condition. So, why did the goalkeeper capture attention in both conditions? First, the goalkeeper takes up a large proportion of the target area (see Figure 1), and he is the only conspicuous and moving stimulus in the environment. Second, it is possible that participants were checking for advance cues as to which way the goalkeeper might move (Kuhn, 1988; Wood \& Wilson, in press). Van der Kamp (2006) has previously suggested that a goalkeeper-focused strategy may be useful if the keeper makes an early decision to dive one way, leaving the other side of the goal unprotected. However, as the goalkeeper did not move until penalties had been struck, this would not appear to be an effective strategy (see performance results).

As nonanxious participants did not consistently adopt a single goal-driven strategy, it is difficult to accurately determine the balance between top-down (goal-driven) and bottom-up (stimulus driven) attentional control. The antisaccade task used by Derakshan et al. (2009) had specific top-down instructions, so differences in top-down and bottom-up control were easily determined. Future studies using a penalty task should therefore explicitly manipulate the top-down control aspect of each penalty kick by varying the payoffs for an optimal strategy. This would enable a stricter definition of what goal-directed control should be for the task, and more effectively create the conflict between salient stimuli and an optimal top-down strategy.

The goal-directed attentional control could be manipulated by creating optimal scoring zones where the incentive is greater (e.g., the top-left corner might be worth 10 points as opposed to 1 point for scoring a goal elsewhere). The importance of aiming toward the goal corner could also be manipulated by penalizing shots that are saved (e.g., -10 points), more than shots that narrowly miss the target (e.g., 0 points). ACT would predict that, as anxiety impairs attentional flexibility at the expense of bottom-up, stimulus-driven control, anxious individuals would be less successful at adjusting their attentional strategies to meet varying top-down objectives.

A final caveat is that other more descriptive attentional theories could possibly explain the findings that heightened anxiety caused attention to be directed centrally to the goalkeeper. First, Wegner's (1994) theory of ironic processing purports that the instruction to avoid a thought or action may ironically increase the tendency to engage in this thought or action, especially when attentional resources are taxed. Bakker et al. (2006) have previously shown that under time pressure, footballers may look toward the goalkeeper despite instructions not to. Second, Easterbrook's (1959) cue utilization hypothesis suggests that increased arousal narrows attention, restricting the range of incidental cues that are used (i.e., the attentional field narrows). Janelle, Singer, and Williams (1999) found support for attention-narrowing effects that were due to heightened anxiety in an auto racing simulation in which participants were less successful at detecting peripherally presented targets when anxious, while central task performance was maintained.

It is difficult to discount these other postulations for this particular task involving a centralized source of threat. However, Easterbrook's predictions have not always been supported (see Eysenck, 1982, for a review), and ACT can be 
considered a more overarching framework for anxiety and attentional biases. Compared with Wegner's theory of ironic processing and Easterbrook's cue utilization hypothesis, ACT is more closely linked to other theoretical developments of anxiety and visual attention (Bar-Haim et al., 2007; Weierich et al., 2008). In addition, as Eysenck et al. (2007) suggest, ACT's predictions are related to a major theoretical approach to attention (Corbetta \& Schulman, 2002), which is not the case for the other two theories. By experimentally manipulating the top-down strategic nature of the task, and the salience of the distracting goalkeeper, as we have already suggested, future studies may provide stronger comparative tests of the predictions of these three attentional control theories.

\section{Performance}

In developing ACT, Eysenck et al. (2007) maintained the prediction from PET that anxiety would have a greater impact on processing efficiency than on performance effectiveness (see Derakshan et al., 2009). In the current study, when gaze was constrained centrally (toward the goalkeeper), shots were also hit more centrally (by a mean of $14 \mathrm{~cm}$ ) and therefore closer within the goalkeeper's reach. Impairments in the efficiency of attentional control (more stimulus driven) therefore resulted in subsequent impairments in performance (accuracy of shots). The findings of the current study therefore reinforce those from Wilson et al.'s (2009) study of basketball free throw shooting and may reflect the critical interdependence between attention and performance in far aiming tasks. These results are also in agreement with those of Wood and Wilson (in press), who explicitly manipulated the gaze of penalty takers and found that resultant shot location was closely linked to final aiming fixation locations. Participants tended to look where they aimed, with centrally focused fixations inducing more central ball placements.

It is important to note that despite this impairment in where the ball was hit, participants were actually reasonably successful at scoring penalties; on only 14 occasions did the goalkeeper make a save ( 8 in the no-threat and 6 in the highthreat condition). The fact that the goalkeeper was prevented from anticipating shot direction is likely to have influenced this result, and, as there was no prize money available to the goalkeeper, so might have motivational factors. However, including shots that missed the target (four in the no-threat, six in the high-threat condition), there was an $86 \%$ success rate across both conditions-similar to the $80 \%$ success rates reported in real-life penalty kicks (Bar-Eli, Azar, Ritov, Keidar-Levin, $\&$ Schein, 2007). Therefore, the performance of the goalkeeper and kickers does not seem completely at odds with what we might expect from more ecologically valid, 11-a-side kicks. In addition, these experienced participants would not have found their success ratio to be unrealistically high, thereby maintaining their levels of uncertainty with regards to goal outcome.

Whereas the task was representative of a 5-a-side penalty kick, care must be taken in applying the findings directly to the performance of 11-a-side penalties. The decision to use a 5-a-side goal was made to provide the strictest possible internal control for testing the predictions of ACT in an applied environment. Because 5-aside penalties do not incorporate a run-up before kicking, differences in run-ups between kickers and in the timing of gaze behavior while running up to the ball did not have to be accounted for. We would expect a more dynamic situation to occur during the run-up in the 11-a-side game, with the penalty taker's attention perhaps switching between the goal target areas, goalkeeper, and the ball during the run-up. 
With a full run-up, the final fixation to the ball before initiating the kicking action (i.e., the quiet eye period; Vickers, 1996) would also likely play a more important role in ensuring an accurate ball strike (see Vickers, 2007). Fixations to the ball were not analyzed in the current study since we were more interested in examining differences in the fixations to the target locations across conditions. As a result of the one-step nature of the shot, fixations to the ball were not critical to ensure accurate contact. Indeed, of the 167 kicks analyzed, on only 32 occasions ( 15 in the low- and 17 in the high-threat condition) did the kicker fixate on the ball while shooting. Therefore, $81 \%$ of shots had no fixation on the ball after an initial check of its placement on the spot, and were executed while the participants looked toward the target area (goal/goalkeeper).

Although more research is required to further our understanding of why performers may miss penalties in evaluative situations, the results found in the current study, along with those of Bakker et al. (2006), van der Kamp (2006), and Wood and Wilson (in press), would suggest that inappropriate attention to the goalkeeper is a major factor. Indeed, recent anecdotal evidence from the top level of the 11-aside game also supports this interpretation. In the 2009 FA Cup semifinal, Dimitar Berbatov (a $£ 30$ million forward for Manchester United) missed a penalty in the sudden death shoot-out by feebly hitting his shot directly at the goalkeeper. He was derided in the press for his poor attempt and rationalized his miss as follows: "I was looking for the goalkeeper and in the last moment he took the angle I was going for, so he saved it" (Berbatov, 2009). If under pressure, players are more likely to find the goalkeeper attention grabbing, a gaze-training intervention designed to direct attention to the target areas of the goal may help maintain a sense of control and reduce uncertainty in these situations. Future research may attempt to test the validity of such training programs for protecting against attentional disruptions that are due to increased anxiety.

To conclude, experienced footballers looked at the goalkeeper significantly earlier and for longer periods when anxious, with these changes in attentional control negatively influencing resultant shot placement. These results are supportive of anxious individuals having an attentional bias toward threatening stimuli, probably owing to a disruption in the balance between the goal-directed and stimulus-driven attentional control systems. These findings add support to the predictions of ACT (Eysenck et al., 2007) in motor task performance under pressure and may offer a mechanistic explanation as to why penalty kicks are missed in pressure environments.

\section{Acknowledgments}

The authors would like to thank Michael Eysenck and two anonymous reviewers for their constructive comments on earlier drafts of this manuscript.

\section{References}

Bakker, F.C., Oudejans, R.D., Binsch, O., \& Van der Kamp, J. (2006). Penalty shooting and gaze behaviour: Unwanted effects of the wish not to miss. International Journal of Sport Psychology, 37, 265-280.

Bar-Eli, M., Azar, O.H., Ritov, I., Keidar-Levin, Y., \& Schein, G. (2007). Action bias among elite goalkeepers: The case of penalty kicks. Journal of Economic Psychology, 28, 606-621. 
Bar-Haim, Y., Lamy, D., Pergamin, L., Bakermans-Kranenburg, M.J., \& van Ijzendoorn, M.H. (2007). Threat-related attentional bias in anxious and nonanxious individuals: A meta-analytic study. Psychological Bulletin, 133, 1-24.

Behan, M., \& Wilson, M. (2008). State anxiety and visual attention: The role of the quiet eye period in aiming to a far target. Journal of Sports Sciences, 26, 207-215.

Berbatov, D. (2009). I let the team down. Retrieved 14th May, from Sky Sports Website: http://www.skysports.com/story/0,19528,11667_5211477,00.html

Cohen, J. (1988). Statistical power analysis for the behavioral sciences (2nd ed.). Hillsdale, NJ: Lawrence Earlbaum Associates.

Corbetta, M., \& Shulman, G.L. (2002). Control of goal-directed and stimulus-driven attention in the brain. Nature Reviews. Neuroscience, 3, 201-215.

Derakshan, N., Ansari, T.L., Hansard, M., Shoker, L., \& Eysenck, M.W. (2009). Anxiety, inhibition, efficiency and effectiveness: An investigation using the antisaccade task. Experimental Psychology, 56, 48-55.

Easterbrook, J.A. (1959). The effect of emotion on cue utilization and the organization of behavior. Psychological Review, 66, 183-201.

Eysenck, M.W. (1982). Attention and arousal: Cognition and performance. Berlin: SpringerVerlag.

Eysenck, M.W., \& Calvo, M.G. (1992). Anxiety and performance: The processing efficiency theory. Cognition and Emotion, 6, 409-434.

Eysenck, M.W., Derakshan, N., Santos, R., \& Calvo, M.G. (2007). Anxiety and cognitive performance: Attentional control theory. Emotion (Washington, D.C.), 7, 336-353.

Fisher, A.C., \& Zwart, E.F. (1982). Psychological analysis of athlete's anxiety responses. Journal of Personality and Social Psychology, 58, 844-854.

Greenlees, I.A., Leyland, A., Thelwell, R., \& Filby, W. (2008). Soccer penalty takers' uniform colour and pre-penalty kick gaze affect the impressions formed of them by opposing goalkeepers. Journal of Sports Sciences, 26, 569-576.

Hill, R.A., \& Barton, R.A. (2005). Red enhances human performance in contests. Nature, 435, 293.

Janelle, C.M. (2002). Anxiety arousal and visual attention: a mechanistic account of performance variability. Journal of Sports Sciences, 20, 237-251.

Janelle, C.M., Singer, R.N., \& Williams, A.M. (1999). External distraction and attentional narrowing: Visual search evidence. Journal of Sport \& Exercise Psychology, 21, 70-91.

Jordet, G. (2009). Why do English players fail in soccer penalty shootouts? A study of team status, self-regulation, and choking under pressure. Journal of Sports Sciences, 27, 97-106.

Jordet, G., Elferink-Gemser, M.T., Lemmink, K.A.P.M., \& Visscher, C. (2006). The "Russian roulette" of soccer?: Perceived control and anxiety in a major tournament penalty shootout. International Journal of Sport Psychology, 37, 281-298.

Jordet, G., Hartman, E., Visscher, C., \& Lemmink, K.A.P.M. (2007). Kicks from the penalty mark in soccer: The roles of stress, skill, and fatigue for kick outcomes. Journal of Sports Sciences, 25, 121-129.

Krane, V. (1994). The mental readiness form as a measure of competitive state anxiety. The Sport Psychologist, 8, 189-202.

Kuhn, W. (1988). Penalty-kick strategies for shooters and goalkeepers. In T. Reilly, A. Lees, K. Davids, \& W.J. Murphy (Eds.), Science and football (pp. 489-492). London: E \& FN Spon.

Lox, C.L. (1992). The role of perceived threat as a cognitive component of state anxiety and confidence. Perceptual and Motor Skills, 75, 1092-1094.

Martens, R., Burton, D., Vealey, R.S., Bump, L., \& Smith, D.E. (1990). Development and validation of the Competitive State Anxiety Inventory-2. In R. Martens, R.S. Vealey, \& D. Burton (Eds.), Competitive anxiety in sport (pp. 117-178). Champaign, IL: Human Kinetics. 
Masters, R.S.W., van der Kamp, J., \& Jackson, R.C. (2007). Imperceptibly off-center goalkeepers influence penalty-kick direction in soccer. Psychological Science, 18, 222-223.

Murray, N.P., \& Janelle, C.M. (2003). Anxiety and performance: A visual search examination of the processing efficiency theory. Journal of Sport \& Exercise Psychology, 25, $171-187$.

Murray, N.P., \& Janelle, C.M. (2007). Event-related potential evidence for the processing efficiency theory. Journal of Sports Sciences, 25, 161-171.

Nieuwenhuys, A., Pijpers, J.R., Oudejans, R.R.D., \& Bakker, F.C. (2008). The influence of anxiety on visual attention in climbing. Journal of Sport \& Exercise Psychology, 30, 171-185.

Van der Kamp, J. (2006). A field simulation study of the effectiveness of penalty kick strategies in soccer: Late alteration of kick direction increase errors and reduce accuracy. Journal of Sports Sciences, 24, 467-477.

Van der Kamp, J., \& Masters, R.S.W. (2008). The human Müller-Lyer illusion in goalkeeping. Perception, 37, 951-954.

Vickers, J.N. (1996). Visual control when aiming at a far target. Journal of Experimental Psychology. Human Perception and Performance, 2, 324-354.

Vickers, J.N. (2007). Perception, cognition and decision training: The quiet eye in action. Champaign, IL: Human Kinetics.

Vickers, J.N., \& Williams, A.M. (2007). Performing under pressure: The effects of physiological arousal, cognitive anxiety, and gaze control in biathlon. Journal of Motor Behavior, 39, 381-394.

Wegner, D.M. (1994). Ironic processes of mental control. Psychological Review, 101, 34-52.

Weierich, M.R., Treat, T.A., \& Hollingworth, A. (2008). Theories and measurement of visual attentional processing in anxiety. Cognition and Emotion, 22, 985-1018.

Williams, A.M., \& Elliott, D. (1999). Anxiety, expertise, and visual search strategy in karate. Journal of Sport \& Exercise Psychology, 21, 326-375.

Williams, A.M., Vickers, J.N., \& Rodrigues, S. (2002). The Effects of Anxiety on Visual Search, Movement Kinematics, and Performance in Table Tennis: A Test of Eysenck and Calvo's Processing Efficiency Theory. Journal of Sport \& Exercise Psychology, 24, 438-455.

Wilson, M. (2008). From processing efficiency to attentional control: A mechanistic account of the anxiety-performance relationship. International Review of Sport and Exercise Psychology, 1, 184-201.

Wilson, M., Smith, N.C., \& Holmes, P.S. (2007a). The role of effort in moderating the anxiety-performance relationship: Testing the conflicting predictions of processing efficiency theory and the conscious processing hypothesis. The British Journal of Psychology, 98, 411-428.

Wilson, M.R., Vine, S.J., \& Wood, G. (2009). The influence of anxiety on visual attentional control in basketball free-throw shooting. Journal of Sport \& Exercise Psychology, $31,152-168$.

Wilson, M., Chattington, M., Marple-Horvat, D.E., \& Smith, N.C. (2007b). A comparison of self-focus versus attentional explanations of choking. Journal of Sport \& Exercise Psychology, 29, 439-456.

Wilson, M., Smith, N.C., Chattington, M., Ford, M., \& Marple-Horvat, D.E. (2006). The role of effort in moderating the anxiety-performance relationship: Testing the predictions of processing efficiency theory in simulated rally driving. Journal of Sports Sciences, $24,1223-1233$.

Wood, G., \& Wilson, M.R. (in press).Gaze behaviour and shooting strategies in football penalty kicks: Implications of a 'keeper-dependent approach. International Journal of Sport Psychology.

Manuscript received: January 22, 2009

Revision accepted: August 29, 2009 